\title{
FireWalk: A Location-Based Walking Game for Health Promoting
}

\author{
Yin-Li Lo, Hao-Han Chen and Hsing Mei ${ }^{+}$ \\ The Department of Computer Science and Information Engineering, Fu Jen Catholic University
}

\begin{abstract}
To maintain a quality life, exercise is considered the most effective way. To avoid stressful exercise, integrate the advantages of exercise and game is possible approach. This research design and develop a location-based game, named FireWalk, to motivate users to exercise. Location-based mobile games are played based on physical location of game players. FireWalk kept exercise record for the user, and evaluate the game experience and exercise experience for each play segment. It is expected to enhance the exercise motivation of players, and thus to promote their health. With the automated play functions of FireWalk, the interrupting effect of exercise and safety concerns of location-based game are considered. Good game experience and exercise experience can be existed at the same time.
\end{abstract}

Keywords: location-based mobile game, health promotion, game exercise, exercise experience

\section{Introduction}

People start exercising to improve health condition [1], [2]. However, exercising only for health is stressful for most people. Hence, extra motivation is required to make exercise more attractive [3]. This research design and develop a location-based mobile game with exercise records and special functions. The goal is to enrich user's exercise experience with game playing.

The most significant characteristics of a location-based game is that the player's physical location will affect the game play [4], [5]. Thus, players may have special sense of Online to Offline mapping [6]. Players will walk and play the game simultaneously [7]. Ingress is a famous location-based game develop by Google Niantic Lab. However, Ingress does not provide functions related exercise record. On the other hand, there are many APPs and services focus on exercise recording, such as Runkeeper. In this paper, a location-based game, named FireWalk, integrated with exercise (Walking/Jogging) record is presented. FireWalk take advantages of both gaming and exercising, and avoid the disadvantages of boring exercise and safety concerns. Beside the common mobile-game features, FireWalk is designed with automated function to enhance the exercise experience and to avoid dangerous condition [8].

To clarify between the exercise experience and game experience of the player, this study designed an experience evaluation model.

The movement in FireWalk is linked with position in real world. Hence, players need to move his location near Portal to interact with. There are three camps in FireWalk, and each marked with different color: green, red, and orange. Portal's color depend on which camp it belongs to. If color is grey, the corresponding portal is currently unclaimed. Players deploy defense items, also called resonator, on portals to acquire them for their camp or help to defend them (as in Fig. 2). A portal with no resonator is unclaimed, and it is free to deploy by player from any camp. If a player want to take over a portal controlled by other camps, a player must attack it with weapon until it is unclaimed. Deploying and attacking need corresponding resource (resonator and weapon), and players can obtain them from hacking or daily reward (as in Fig. 3). A player can hack any portal and receive resource, subject to limitation of following rule:

\footnotetext{
${ }^{+}$Corresponding author. Tel.: +886-2-29053880; fax: + 886-2-29023550.
}

E-mail address: $\{$ francislee, kk121155\}@weco.net, mei@csie.fju.edu.tw. 
Players cannot hack same portal again in 5 minutes. Daily reward can be receive once a day, and the purpose of it is to encourage players coming back to play FireWalk every day. Interaction statistics are recorded as game achievement (as in Fig. 4). Players can either compete to other players or themselves.

Consider gaming and exercising parameters while play FireWalk, Game Experience Index (GEI) and Exercise Experience Index (EEI) are designed. User can play the game with or without automated functions, to get difference level of satisfaction. This paper introduce about the design of the equations, the definition of the index and the examples of above in detail in section III.

The rest of the paper is organized as follows. In next section, the game rules and automated functions of FireWalk game are introduced. Section III presents the evaluation model, experience index, and experiment design. A conclusion and the future research are presented in Section IV.

\section{Procedure for Paper Submission}

FireWalk is a cross-platform location-based game developed with HTML5. Currently, FireWalk is a subservice in Fitness Promotion Space that hosted on Joyful Health Space. The main goal of Fitness Promotion Space is to help users maintain their exercise record and promote their health condition. Whereas FireWalk Game make exercise more fun and motivate users to do more exercise.

The reason that FireWalk make exercise more effective is the integration of game and exercise record. The game in FireWalk is inspired by Ingress [9]. The game rules will be presented in Section II.A. The exercise record in FireWalk is inspired by Runkeeper [10] and we present the detail in Section II.B. FireWalk integrated game with exercise record and link them together, which means that exercise record will be the parameters in game and playing game can accumulate exercise records. Hence, FireWalk encourage users to perform more exercise.

The two major problems of location-based game are: (1) the interrupting of continuing exercise; and (2) safety concern on game and exercise at same time. Hence, we design and develop the automated functions for the FireWalk, which avoid the interrupting effect of exercise and provide a safer exercise environment.

Fig. 1 shows the game snapshot of FireWalk. Each flag-shape marker is a FireWalk Portal. Different colors of Portal mean different camps in game. The circle marker is player's current location. In this figure, we can see that player is located at University Auditorium, Fu Jen Catholic University. On the top-left of figure, the player's level and total distance are shown. The functions menu is presented on the top-right corner. Fig. 2 introduces the portal details. Fig. 3(a) shows the daily reward, and Fig. 3(b) shows the game achievement.

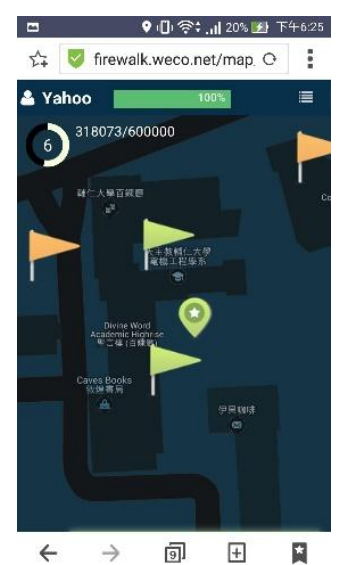

Fig. 1: FireWalk game snapshot.

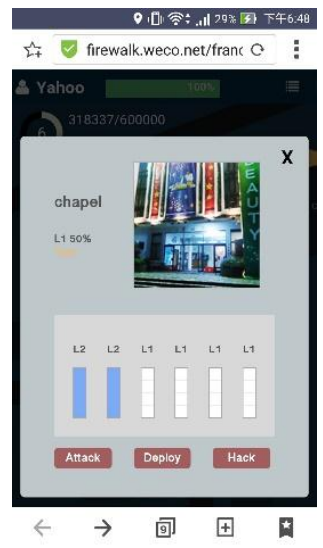

Fig. 2: Portal information: portal location, status of six resonators, actions (attack, deploy, and hack).



(a)

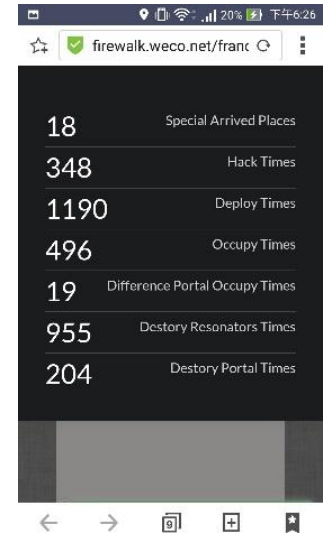

(b)
Fig. 3: Daily reward and Game achievement, including: number of hack action, number of resonator deployed.

The exercise record in FireWalk includes the route, time, distance and so on.. Firewalk not only display the result in realtime, but also present monthly exercise statistics with chart. Fig. 4 is a real time exercise 
record snapshot. The red line on map is the route, and the black area above shows the exercise time, distance, and average speed. Fig. 5 illustrates monthly exercise statistic. It shows total number of exercise time, exercise distance, and consumed calorie. Whereas it present the everyday total of exercise time, exercise distance, and consumed calorie with chart.

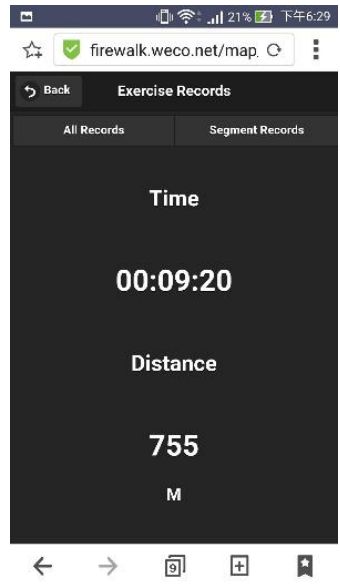

(a)

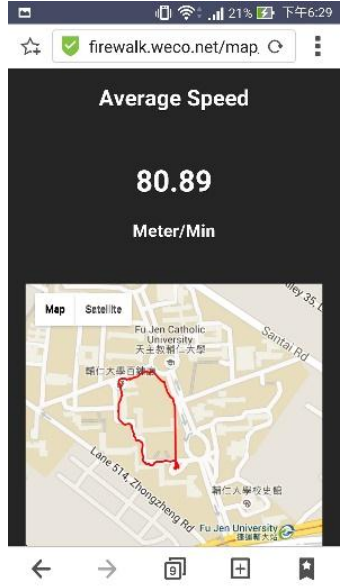

(b)

Fig. 4: Real time exercise record.

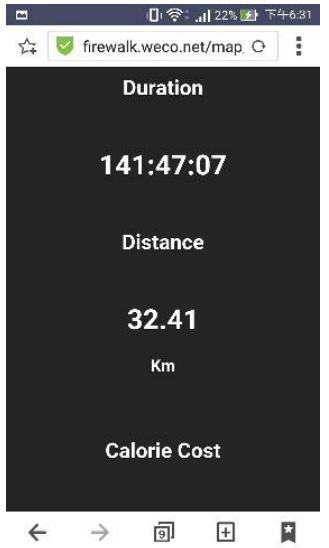

(a)

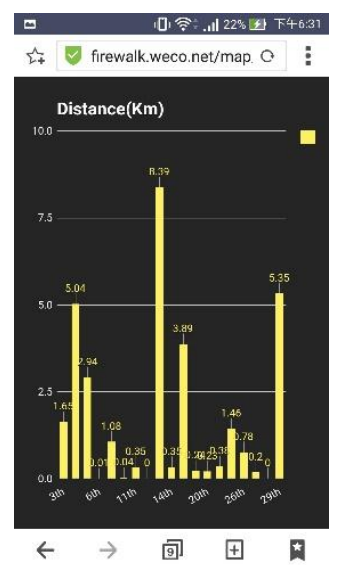

(b)

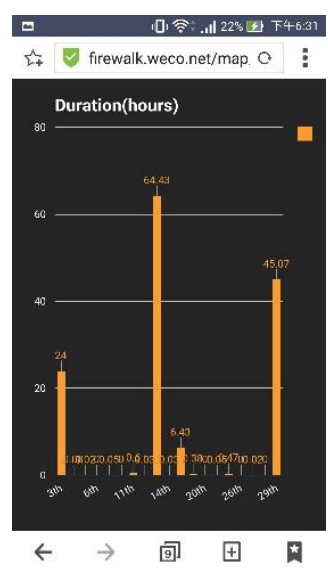

(c)

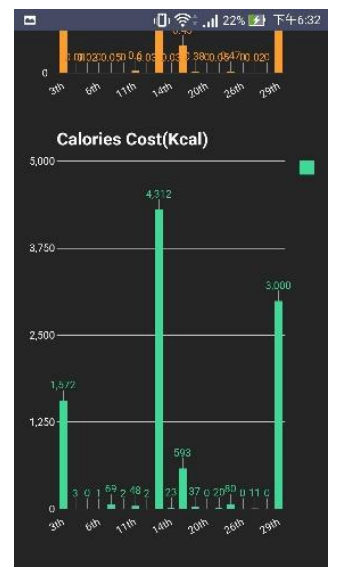

(d)

Fig. 5: Monthly exercise statistics.

\subsection{Play automation}

The unique feature of location-based game is the user's physical location will affect the game play. However, this type of game have a major problem that player have to watch smartphone while playing. Therefore, the interruption of exercise cause poor exercise experience. Even more serious, some gameplay activities may cause the safety concerns. To encounter with this problem, we design the automated play functions for users who like to focus on exercise. Whenever players feel poor exercise experience, or in inconvenient areas, such as road, automated functions could be selected to replace the human player.

When using the automated functions, players can use the default setting or configure it to meet their own needs. The idea of default setting for automated deploy is to be resource saving. Fig. 6 shows an automated function setting snapshot. The system will automated deploy the type of item that user owned most. As to the setting of automated attack is based on the currently energy level of resonator to choose the weapon. The most cost effective weapon will be selected to attack. The strong attack/deploy setting causes the selection of the most powerful attacking/deploying items. Similarly, the weak attack/deploy setting causes the attacking/deploying with the weakest item.

\section{Game and Exercise Experience Index}


In order to evaluate the impact of game automation to the players, we model the game experience and exercise experience with quantified values. Two indexes are designed to evaluate the achievement of the game playing: the Game Experience Index(GEI); and the Exercise Experience Index(EEI). GEI and EEI were calculated for each playing period (i.e. game segment). In the next 2 sub-sections, definitions of GEI and EEI are illustrated in details.

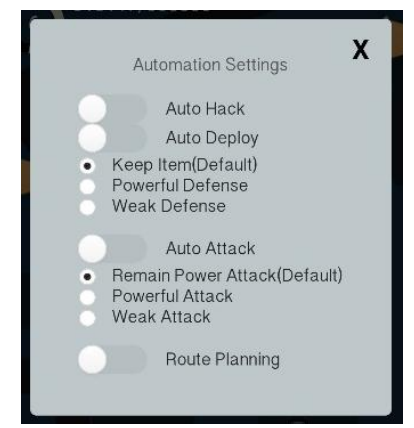

Fig. 6. Automated function setting.

\subsection{Game experience index}

The following is the equation of GEI:

$$
\mathrm{GEI}=\sum_{i=1}^{n}\left(T_{i} * W_{i}\right) / \sum_{i=1}^{n}\left(D_{t i} * W_{i}\right) * 100
$$

GEI : Game Experience Index

$n$ : Total number of the tasks in this game segment

$i$ : Task category

$T_{i}$ : Number of tasks of task category $i$.

$W_{i}$ : Assigned weight for category $i$ task

$D_{t i}$ : Default number of tasks in task category $i$ (to have full game experience)

FireWalk game assume the difficulty of the game is proportional to the number of the player's actions. More difficult game actions challenge the player more, and induce better experience. A task consist of one or

more actions. Six categories of player tasks are designed in FireWalk, namely: Hack; Deploy; Occupy portal; Destroy portal; and Destroy resonators. The Game Experience Index (GEI) is defined in (1). More difficult task will get higher weight. The following is the detailed illustration of the above: 1 independent action from player weight 1 , that is, if a task need one action to complete, the task's weight is 1 . If a task needs 2 actions, then the task weight 2. For example, hacking a portal just need to click "hack" to complete, so the weight of this task is 1 . To destroy the portal requires destroying all the resonators. There's 6 resonators per portal. As to destroying the portal is considered equivalent to dis-deploying 6 resonators, the weight of "destroy portal" is set to be 6 .

Equation (1) can be further adjusted to reasonable range, that is, GEI will between 0 and 100 . However, once user exceed the expectation of original game design, GEI could be greater than 100 . The denominator of this equation denote the highest expectation of the game. If a player reach this highest expectation, he will feel satisfied and GEI will be 100 .

\subsection{Exercise experience index}

The following is the equation of EEI:

$$
\mathrm{EEI}=\frac{S_{a}}{S_{d}} * \frac{T_{e}}{T_{t}} * 100
$$

EEI: Exercise Experience

$S_{a}$ : Average speed of this game segment

$S_{d}$ : Default exercise speed (to have full exercise experience) 


\section{$T_{e}$ : Exercise time in this game segment}

$T_{t}$ : Time used in this game segment

This research assumed that faster walking speed and higher proportion of exercise time cause better exercise experience in the game segment. Based on this assumption, Exercise Experience Index (EEI) is designed and the common value will be less than 100. $S_{d}$ is an assigned default value of maximum speed. If user speed reach $S_{d}$, user get the full exercise experience in speed portion of EEI. $S_{d}$ should be adjusted for each individual player. For instance, If the player intends to have intensive training, the $S_{d}$ should be set up with higher default value.

As to second part (Time portion) of EEI, the influence of game interrupting during game play is considered. Player stay at one location for more than 5 seconds is considered as stop. Equation (2) shows the influence of stop and strength of exercise for one play segment. If user's average speed is higher than default exercise speed, and the user did not stop, EEI will over 100.

\subsection{The influence of game automation}

This section describe about experiment in this paper. This experiment is divided to four sections to discuss: 1. do not use game automation, and do not use route planning. 2. Use game automation, but do not use route planning. 3. Do not use game automation, but use route planning. 4. Use game automation and use route planning. Game automation is designed to resolve the problem that play the game will influence exercise, and route planning is designed to improve user's game experience. The final target of this experiment is make player satisfied in both game experience and exercise experience.

\subsection{Experimental verification}

To verify the game experience and exercise experience evaluation model, experiments are designed. We recruited 10 testing players, and the experiment is scheduled begin in March 2016. The assumptions of experiment are:

- Player need to brisk walking while playing the game.

- Player cannot walk back.

- Player has sufficient amount of attacking and defending items.

To distinguish the difference between game experience and exercise experience, each test subject must finish four play segments in FireWalk.

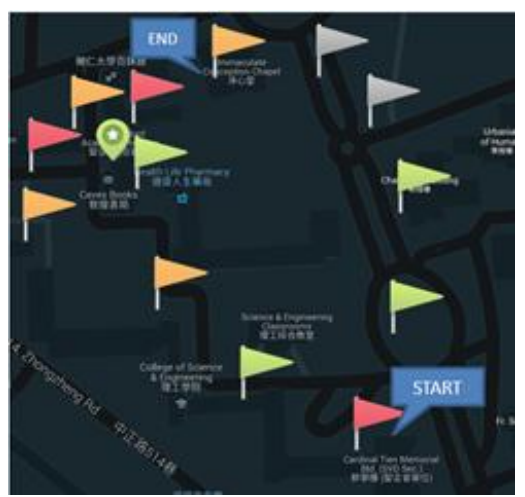

Fig. 7: Experiment environment.

- Play without game automation, and no route planning

In this play segment, user will not use any assistance from game system (i.e. game automation, and route planning). After user leave the START point (in Fig. 7), user play and walk forward to reach the END point.

Expected result: This play segment is control group that without any help from the FireWalk automation function. Good exercise experience is not expected in this case.

- $\quad$ Play with game automation, and without route planning 
In this play segment, user play FireWalk with game automation function. After user leave START point, user choose the forward direction by his/her own free will toward the END point.

Expected result: Because of game automation, user will not be interrupted. Hence, the result EEI should be higher than segment 1).

- Play with route planning, but no game automation

In this play segment, user use route planning function of FireWalk. After leaving START point, player will follow the route tips to play toward the END point.

Expected result: Route planning make user exercise smoothly in game, and higher GEI than segment 1) is expected.

- Play with game automation, and also route planning

After started from START, follow the tips of direction route planning give, and walk toward the END point.

Expected result: Because of the effects from game automation and route planning, player is expected to have both good game and exercise experience.

\section{Conclusion and Future Work}

This paper introduced a location-based game, FireWalk, which integrated game and exercise records. There are three camps in game, and interactions between camps make game more interested. Besides, FireWalk is designed with game reward system to make game more challenging. Concerning health-related functions, FireWalk provide the real time exercise status and monthly exercise statistics. Hence, FireWalk also help users to realize their exercise condition and promote health.

FireWalk is designed with automated function to encounter the safety concern of location-based mobile game. By automation, interrupting and hitting obstacles are less likely to occur while playing. By route planning function, user can follow a route which can reach higher EEI. Both the exercise experience and game experience can be balanced for each user, and user will be satisfied. In the future, we will conduct the real world experiment to verify the game and exercise evaluation model. GEI and EEI are expected to use on the other location-based games and services.

\section{References}

[1] Walker, J.M., and et al., "Effects of exercise on sleep," Journal of Applied Physiology, Vol. 44, No. 6, pp. 945-951, 1987

[2] Blair, Steven N., Yiling Cheng, and J. Scott Holder., "Is physical activity or physical fitness more important in defining health benefits?," Medicine and science in sports and exercise vol. 33, no. 6, pp. 379-399, 2001.

[3] Richard, M., et al. "Intrinsic motivation and exercise adherence," Int J Sport Psychol vol. 28 no. 4, pp. 35-354, 1997.

[4] Nicklas, Daniela, Christoph Pfisterer, and Bernhard Mitschang, "Towards location-based games," Proceedings of the international conference on applications and development of computer games in the 21 st century: ADCOG. Vol. 21, pp.61-67, 2001.

[5] Avouris, Nikolaos M., and Nikoleta Yiannoutsou, "A Review of Mobile Location-based Games for Learning across Physical and Virtual Spaces," J. UCS, Vol. 18, No. 15, pp. 2120-2142, 2012.

[6] G. Treu, J. Martens, M. Schicker, M. Breisinger, and A. Küpper, "Vs Golf - Developing Location-based MultiPlayer Games," Proceedings of the 31st Annual International Computer Software and Applications Conference, Vol. 2, pp. 109-114, July 2007.

[7] A. Laikari, "Exergaming - gaming for health: A bridge between real world and virtual communities," in Consumer Electronics, 2009. ISCE '09. IEEE 13th International Symposium on, pp. 665 -668, May 2009.

[8] João Tiago Pinheiro Neto Jacob and António Fernando Coelho, "Issues in the Development of Location-Based Games," International Journal of Computer Games Technology, vol. 2011, Article ID 495437, 2011.

[9] Ingress.com, (2016). Ingress. [online] Available at: https://www.ingress.com/ [Accessed 1 Mar. 2016], 2016. 
[10] Runkeeper.com, (2016). Runkeeper - Track your runs, walks and more with your iPhone or Android phone. [Online] Available at: https://runkeeper.com/ [Accessed 1 Mar. 2016], 2016.

[11] Melanson Jr, EDWARD L., and PATTY S. Freedson. "Validity of the Computer Science and Applications, Inc.(CSA) activity monitor." Medicine and Science in Sports and Exercise, Vol. 27, No. 6, pp. 934-940, 1995. 